\title{
Random Distributed Feedback Fiber Laser
}

\author{
Sergei K. Turitsyn, Sergey A. Babin, Atalla E. El-Taher, Paul Harper, Dmitriy V. Churkin, \\ Sergey I. Kablukov, Juan Diego Ania-Castañón, Vassilis Karalekas and Evgenii V. Podivilov
}

A basic laser scheme normally requires two key elements: A gain material that provides amplification and an optical cavity that traps the light, creating positive feedback. Lasing occurs when the total gain in the cavity overcomes the total cavity loss. Operational characteristics of conventional lasers are determined both by the distinctive features of the gain medium and by the cavity design that defines the structure of laser modes.

In random lasers with no cavity (or with an open cavity), ${ }^{1,2}$ the output characteristics are determined by the buildup of radiation due to multiple scattering in the gain medium, resulting in randomly embedded local spatial modes that may coexist with non-localized extended modes. ${ }^{3}$ Random lasers have advantages, such as simple technology that does not require a precise microcavity, and low production cost. However, the properties of their output radiation are rather special in comparison to those of conventional lasers, and they are usually characterized by complex features in the spatial, spectral and time domains.

We demonstrated a new type of one-dimensional laser with random distributed feedback based on Rayleigh scattering (RS), which is present in any transparent glass medium due to natural inhomogeneities of refractive index. ${ }^{4}$ The cylindrical fiber waveguide geometry provides transverse confinement, while the cavity is open in the longitudinal direction and does not include any regular point-action reflectors.

Though Rayleigh backscattering is extremely weak, the effect may be accumulated and amplified in the long fiber. Using stimulated Raman scattering to provide distributed amplification, we demonstrate random lasing in low-cost, open-cavity standard transmission fiber with stationary narrowband output power of about $300 \mathrm{~mW}$ from two fiber

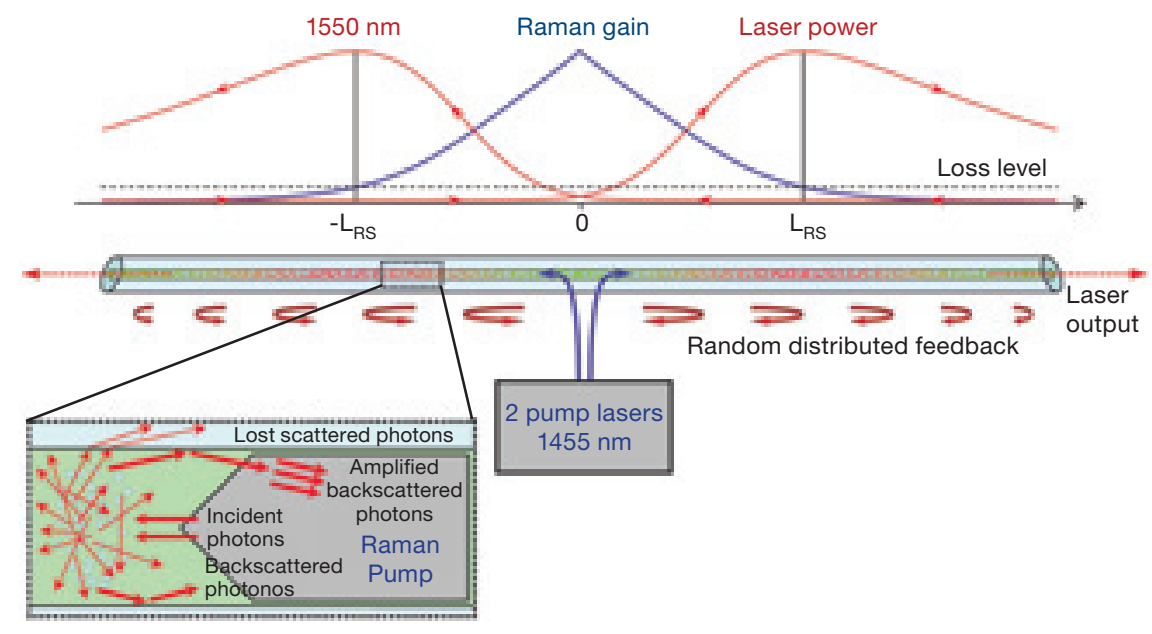

Principle of random distributed feedback fiber laser operation. Photons propagating in a long fiber are coherently scattered in a $83-\mathrm{km}$ fiber by random refractive-index inhomogeneities complying with Rayleigh's law. Most of the scattered photons leak out of the fiber core. Only $Q \sim 10^{-3}$ of them are backscattered and guided by the fiber. Two pump waves coupled at $z=0$ provide distributed Raman gain along the fiber. The backscattered guided photons can be amplified if total gain is larger than the loss level, which is fulfilled for all points $|z|<L_{R S}$. As a result, two forward and backward propagating waves are generated. The numerically calculated laser power distribution (red) and Raman gain (blue) are shown.

ends. The weakness of the RS-based random distributed feedback makes the operation and properties of the demonstrated lasers profoundly different from those of both traditional random lasers and conventional fiber lasers.

Note that RS might also have a critical impact on performances of conventional (with point reflectors) fiber lasers with a long cavity. In particular, the mode structure of Raman fiber lasers with linear cavity formed by highly reflecting mirrors/gratings is washed out at a resonator length of about $300 \mathrm{~km} .^{5}$

In conclusion, the lasing provided by weak random distributed feedback in an amplifying fiber waveguide medium constitutes a new class of laser-the random distributed feedback fiber laser. We believe that new fundamental science as well as new applications and technologies, in particular, for telecommunications and sensing, will emerge as a result of our development. $\triangle$

Sergei K. Turitsyn (s.K.turitsyn@aston.ac.uk), Atalla E. El-Taher, Paul Harper, Juan Diego Ania-Castañón, and Vassilis Karalekas are with the Photonics Research Group, Aston University, Birmingham, United Kingdom. Ania-Castañón is also with the Instituto de Óptica, CSIC, in Madrid, Spain. Sergey A. Babin, Dmitriy V. Churkin, Sergey I. Kablukoy and Evgenii V. Podivilov are with the Institute of Automation and Electrometry, SB RAS, Novosibirsk, Russia.

\section{References}

1. H. Cao. J. Phys. A: Math. Gen. 38, 10497-535 (2005).

2. D.S. Wiersma. Nature Physics 4, 359-67 (2008).

3. J. Fallert et al. Nature Photonics 3, 279-82 (2009).

4. S.K. Turitsyn et al. Nature Photonics, 4, $231-5$ (2010).

5. S.K. Turitsyn et al. Phys. Rev. Lett. 103, 133901 (2009). 